\title{
A Comparison of Random Walks in Dependent Random Environments
}

\author{
Werner R.W. Scheinhardt \\ University of Twente \\ w.r.w.scheinhardt@utwente.nl
}

\author{
Dirk P. Kroese \\ The University of Queensland \\ kroese@maths.uq.edu.au
}

April 13, 2015

\begin{abstract}
We provide exact computations for the drift of random walks in dependent random environments, including $k$-dependent and moving average environments. We show how the drift can be characterized and evaluated using Perron-Frobenius theory. Comparing random walks in various dependent environments, we demonstrate that their drifts can exhibit interesting behavior that depends significantly on the dependency structure of the random environment.
\end{abstract}

MSC subject classifications: Primary 60K37, 60G50; secondary 82B41

Keywords: random walk, dependent random environment, drift, PerronFrobenius eigenvalue

\section{Introduction}

Random walks in random environments (RWREs) are well-known mathematical models for motion through disorganized (random) media. They generalize ordinary random walks whereby the transition probabilities from any position are determined by the random state of the environment at that position. RWREs exhibit interesting and unusual behavior that is not seen in ordinary random walks. For example, the walk can tend to infinity almost surely, while its overall drift is 0 . The reason for such surprising behavior is that RWREs can spend a long time in (rare) regions from which it is difficult to escape - in effect, the walker becomes "trapped" for a long time.

Since the late 1960s a vast body of knowledge has been built up on the behavior of RWREs. Early applications can be found in Chernov [4] and Temkin [17]; see also Kozlov [9] and references therein. Recent applications to charge transport in designed materials are given in Brereton et al. [3] and Stenzel et al. [15]. The mathematical framework for one-dimensional RWREs in independent environments was laid by Solomon [14], and was further extended by Kesten et al. [8], Sinai [13], 
Greven and Den Hollander [6]. Markovian environments were investigated in Dolgopyat [5] and Mayer-Wolf et al. [10]. Alili [1] showed that in the one-dimensional case much of the theory for independent environments could be generalized to the case where the environment process is stationary and ergodic. Overviews of the current state of the art, with a focus on higher-dimensional RWREs, can be found, for example, in Hughes [7], Sznitman [16], Zeitouni [18, 19], and Révész [11].

Although from a theoretical perspective the behavior of one-dimensional RWREs is well understood, from an applied and computational point of view significant gaps in our understanding remain. For example, exact drift computations and comparisons (as oposed to comparisons using simulation) between dependent random environments seem to be entirely missing from the literature. The reason is that such exact computations are not trivial and require additional insights.

The contribution of this paper is twofold. First, we provide new methodology and explicit expressions for the computation of the drift of one-dimensional random walks in various dependent environments, focusing on so-called 'swap models'. In particular, our approach is based on Perron-Frobenius theory, which allows easy computation of the drift and as well as various cutoff points for transient/recurrent behavior. Second, we compare the drift behavior between various dependent environments, including moving average and $k$-dependent environments. We show that this behavior can deviate considerably from that of the (known) independent case.

The rest of the paper is organized as follows. In Section 2 we formulate the model for a one-dimensional RWRE in a stationary and ergodic environment and review some of the key results from [1]. We then formulate a flexible mechanism for constructing dependent random environment that includes the iid, Markovian, $k$-dependent, and moving average environments. In Section 3 we prove explicit (computable) results for the drift for each of these models, and compare their behaviors. Conclusions and directions for future research are given in Section 4 .

\section{Model and preliminaries}

In this section we review some key results on one-dimensional RWREs and introduce the class of 'swap-models' that we will study in more detail.

\subsection{General theory}

Consider a stochastic process $\left\{X_{n}, n=0,1,2, \ldots\right\}$ with state space $\mathbb{Z}$, and a stochastic "Underlying" environment $\mathbf{U}$ taking values in some set $\mathcal{U}^{\mathbb{Z}}$, where $\mathcal{U}$ is the set of possible environment states for each site in $\mathbb{Z}$. We assume that $\mathbf{U}$ is stationary 
(under $\mathbb{P}$ ) as well as ergodic (under the natural shift operator on $\mathbb{Z}$ ). The evolution of $\left\{X_{n}\right\}$ depends on the realization of $\mathbf{U}$, which is random but fixed in time. For any realization $\mathbf{u}$ of $\mathbf{U}$ the process $\left\{X_{n}\right\}$ behaves as a simple random walk with transition probabilities

$$
\begin{aligned}
& \mathbb{P}\left(X_{n+1}=i+1 \mid X_{n}=i, \mathbf{U}=\mathbf{u}\right)=\alpha_{i}(\mathbf{u}) \\
& \mathbb{P}\left(X_{n+1}=i-1 \mid X_{n}=i, \mathbf{U}=\mathbf{u}\right)=\beta_{i}(\mathbf{u})=1-\alpha_{i}(\mathbf{u}) .
\end{aligned}
$$

The theoretical behavior of $\left\{X_{n}\right\}$ is well understood, as set out in the seminal work of Solomon [14]. In particular, Theorems 2.1 and 2.2 below completely describe the transience/recurrence behavior and the Law of Large Numbers behavior of $\left\{X_{n}\right\}$. We follow the notation of Alili [1] and first give the key quantities that appear in these theorems. Define

$$
\sigma_{i}=\sigma_{i}(\mathbf{u})=\frac{\beta_{i}(\mathbf{u})}{\alpha_{i}(\mathbf{u})}, \quad i \in \mathbb{Z},
$$

and let

$$
S=1+\sigma_{1}+\sigma_{1} \sigma_{2}+\sigma_{1} \sigma_{2} \sigma_{3}+\cdots
$$

and

$$
F=1+\frac{1}{\sigma_{-1}}+\frac{1}{\sigma_{-1} \sigma_{-2}}+\frac{1}{\sigma_{-1} \sigma_{-2} \sigma_{-3}}+\cdots
$$

Theorem 2.1. (Theorem 2.1 in [1])

1. If $\mathbb{E}\left[\ln \sigma_{0}\right]<0$, then almost surely $\lim _{n \rightarrow \infty} X_{n}=\infty$.

2. If $\mathbb{E}\left[\ln \sigma_{0}\right]>0$, then almost surely $\lim _{n \rightarrow \infty} X_{n}=-\infty$.

3. If $\mathbb{E}\left[\ln \sigma_{0}\right]=0$, then almost surely $\liminf _{n \rightarrow \infty} X_{n}=-\infty$ and $\limsup _{n \rightarrow \infty} X_{n}=\infty$.

Theorem 2.2. (Theorem 4.1 in [1])

1. If $\mathbb{E}[S]<\infty$, then almost surely $\lim _{n \rightarrow \infty} \frac{X_{n}}{n}=\frac{1}{\mathbb{E}\left[\left(1+\sigma_{0}\right) S\right]}=\frac{1}{2 \mathbb{E}[S]-1}$.

2. If $\mathbb{E}[F]<\infty$, then almost surely $\lim _{n \rightarrow \infty} \frac{X_{n}}{n}=\frac{-1}{\mathbb{E}\left[\left(1+\sigma_{0}^{-1}\right) F\right]}=\frac{-1}{2 \mathbb{E}[F]-1}$.

3. If $\mathbb{E}[S]=\infty$ and $\mathbb{E}[F]=\infty$, then almost surely $\lim _{n \rightarrow \infty} \frac{X_{n}}{n}=0$.

Note that we have added the second equalities in statements 1 . and 2. of Theorem 2.2. These follow directly from the stationarity of $\mathbf{U}$.

We will call $\lim _{n \rightarrow \infty} X_{n} / n$ the drift of the process $\left\{X_{n}\right\}$, and denote it by $V$. Note that, as mentioned in the introduction, it is possible for the chain to be transient with drift 0 (namely when $\mathbb{E}\left[\ln \sigma_{0}\right] \neq 0, \mathbb{E}[S]=\infty$ and $\mathbb{E}[F]=\infty$ ). 


\section{$2.2 \quad$ Swap model}

We next focus on what we will call swap models, as studied by Sinai [13]. Here, $\mathcal{U}=\{-1,1\}$; that is, we assume that all elements $U_{i}$ of the process $\mathbf{U}$ take value either -1 or +1 . We assume that the transition probabilities in state $i$ only depends on $U_{i}$ and not on other elements of $\mathbf{U}$, as follows. When $U_{i}=-1$, the transition probabilities of $\left\{X_{n}\right\}$ from state $i$ to states $i+1$ and $i-1$ are swapped with respect to the values they have when $U_{i}=+1$. Thus, for some fixed value $p$ in $(0,1)$ we let $\alpha_{i}(\mathbf{u})=p\left(\right.$ and $\left.\beta_{i}(\mathbf{u})=1-p\right)$ if $u_{i}=1$, and $\alpha_{i}(\mathbf{u})=1-p\left(\right.$ and $\beta_{i}(\mathbf{u})=p$ ) if $u_{i}=-1$. Thus, (2.1) becomes

$$
\mathbb{P}\left(X_{n+1}=i+1 \mid X_{n}=i, \mathbf{U}=\mathbf{u}\right)= \begin{cases}p & \text { if } u_{i}=1 \\ 1-p & \text { if } u_{i}=-1\end{cases}
$$

and

$$
\mathbb{P}\left(X_{n+1}=i-1 \mid X_{n}=i, \mathbf{U}=\mathbf{u}\right)= \begin{cases}1-p & \text { if } u_{i}=1 \\ p & \text { if } u_{i}=-1\end{cases}
$$

Next, we choose a dependence structure for $\mathbf{U}$ using the following simple, but novel, construction. Let $\left\{Y_{i}, i \in \mathbb{Z}\right\}$ be a stationary and ergodic Markov chain taking values in some finite set $M$ and let $g: M \rightarrow\{-1,1\}$ be a given function. Now define the environment at state $i$ as $U_{i}=g\left(Y_{i}\right), i \in \mathbb{Z}$. Despite its simplicity, this formalism covers a number of interesting dependence structures on $\mathbf{U}$, discussed next.

iid environment. In this case the $\left\{U_{i}\right\}$ are i.i.d. random variables, with $\alpha \stackrel{\text { def }}{=}$ $\mathbb{P}\left(U_{i}=1\right)=1-\mathbb{P}\left(U_{i}=-1\right)$. Formally, this fits the framework above by choosing $g$ the identity function on $M=\{-1,1\}$ and $\left\{Y_{i}\right\}$ the Markov chain with one-step transition probabilities $\mathbb{P}\left(Y_{i}=1 \mid Y_{i-1}=-1\right)=\mathbb{P}\left(Y_{i}=1 \mid Y_{i-1}=1\right)=\alpha$ for all $i$.

$k$-dependent environment. Define a $k$-dependent environment as an environment $\left\{U_{i}\right\}$ for which

$$
\begin{aligned}
& \mathbb{P}\left(U_{i}=u_{i} \mid U_{i-1}=u_{i-1}, U_{i-2}=u_{i-2}, \ldots\right)= \\
& \mathbb{P}\left(U_{i}=u_{i} \mid U_{i-1}=u_{i-1}, U_{i-2}=u_{i-2}, \ldots, U_{i-k+1}=u_{i-k+1}\right), u_{j} \in\{-1,1\} .
\end{aligned}
$$

Special cases are the independent environment ( $k=0$; see above) and the so-called Markovian environment $(k=1)$. For $k \geqslant 1$, let $\left\{Y_{i}, i \in \mathbb{Z}\right\}$ be a Markov chain that takes values in $M=\{-1,1\}^{k}$ such that from any state $\left(u_{i-k}, \ldots, u_{i-1}\right)$ only two possible transitions can take place, given by

$$
\left(u_{i-k}, \ldots, u_{i-1}\right) \rightarrow\left(u_{i-k+1}, \ldots, u_{i-1}, u_{i}\right), \quad u_{i} \in\{-1,1\}
$$


with corresponding probabilities $1-a_{\left(u_{i-k}, \ldots, u_{i-2}\right)}, a_{\left(u_{i-k}, \ldots, u_{i-2}\right)}, b_{\left(u_{i-k}, \ldots, u_{i-2}\right)}$, and $1-b_{\left(u_{i-k}, \ldots, u_{i-2}\right)}$, for $\left(u_{i-1}, u_{i}\right)$ equal to $(-1,-1),(-1,1),(1,-1)$, and $(1,1)$, respectively. Now define $U_{i}$ as the last component of $Y_{i}$. Then $\left\{U_{i}, i \in \mathbb{Z}\right\}$ is a $k$-dependent environment, and $Y_{i}=\left(U_{i-k+1}, \ldots, U_{i}\right)$. In the special case $k=1$ (Markovian environment), we omit the subindices of $a$ (transition probability from $U_{i-1}=-1$ to $\left.U_{i}=+1\right)$ and $b$ (from $U_{i-1}=+1$ to $U_{i}=-1$ ).

Moving average environment. Consider a "moving average" environment, which is built up in two phases as follows. First, start with an iid environment $\left\{\widehat{U}_{i}\right\}$ as in the iid case, with $\mathbb{P}\left(\widehat{U}_{i}=1\right)=\alpha$. Let $Y_{i}=\left(\widehat{U}_{i}, \widehat{U}_{i+1}, \widehat{U}_{i+2}\right)$. Hence, $\left\{Y_{i}\right\}$ is a Markov process with states $1=(-1,-1,-1), 2=(-1,-1,1), \ldots, 8=(1,1,1)$ (lexicographical order). The corresponding transition matrix is given by

$$
P=\left[\begin{array}{cccccccc}
1-\alpha & \alpha & 0 & 0 & 0 & 0 & 0 & 0 \\
0 & 0 & 1-\alpha & \alpha & 0 & 0 & 0 & 0 \\
0 & 0 & 0 & 0 & 1-\alpha & \alpha & 0 & 0 \\
0 & 0 & 0 & 0 & 0 & 0 & 1-\alpha & \alpha \\
1-\alpha & \alpha & 0 & 0 & 0 & 0 & 0 & 0 \\
0 & 0 & 1-\alpha & \alpha & 0 & 0 & 0 & 0 \\
0 & 0 & 0 & 0 & 1-\alpha & \alpha & 0 & 0 \\
0 & 0 & 0 & 0 & 0 & 0 & 1-\alpha & \alpha
\end{array}\right] .
$$

Now define $U_{i}=g\left(Y_{i}\right)$, where $g\left(Y_{i}\right)=1$ if at least two of the three random variables $\widehat{U}_{i}, \widehat{U}_{i+1}$ and $\widehat{U}_{i+2}$ are 1 , and $g\left(Y_{i}\right)=-1$ otherwise. Thus,

$$
(g(1), \ldots, g(8))=(-1,-1,-1,1,-1,1,1,1),
$$

and we see that each $U_{i}$ is obtained by taking the moving average of $\widehat{U}_{i}, \widehat{U}_{i+1}$ and $\widehat{U}_{i+2}$, as illustrated in Figure 2.2.

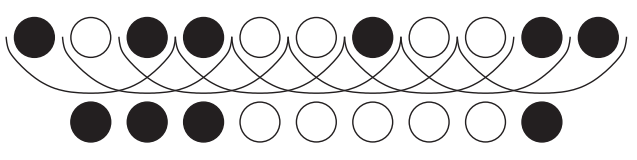

Figure 1: Moving average environment.

\section{Evaluating the drift}

In this section we first give the general solution approach for the Markov-based swap model, and then further specify the transience/recurrence and drift results to the Markov environment, the 2-dependent environment, and the moving average environments. We omit a separate derivation for the i.i.d. environment, which can be viewed as a special case of the Markovian environment, see Remark 3.1. 


\subsection{General solution for swap models}

Due to the choice of notation for the states in $\mathcal{U}=\{-1,1\}$ we can, for any swap model, write $\sigma_{i}$ (defined in $\left.(2.2)\right)$ as:

$$
\sigma_{i}=\frac{p}{1-p} \mathbb{I}\left(U_{i}=-1\right)+\frac{1-p}{p} \mathbb{I}\left(U_{i}=1\right)=\sigma^{U_{i}},
$$

where $\sigma=(1-p) / p$. Consequently, for the key quantity in Theorem 2.1 we find:

$$
\mathbb{E}\left[\ln \sigma_{0}\right]=\mathbb{E}\left[U_{0} \ln \sigma\right]=\ln \sigma \mathbb{E}\left[U_{0}\right]
$$

the sign of which (and hence the a.s. limit of $X_{n}$ ) only depends on whether $p$ is less than or greater than $1 / 2$, and on whether $\mathbb{E}\left[U_{0}\right]$ is positive or negative, regardless of the dependence structure between the $\left\{U_{i}\right\}$.

Furthermore, for the key quantities in Theorem 2.2 we have:

$$
\mathbb{E}[S]=\sum_{n=0}^{\infty} \mathbb{E}\left[\sigma^{\sum_{i=1}^{n} U_{i}}\right] \quad \text { and } \quad \mathbb{E}[F]=\sum_{n=0}^{\infty} \mathbb{E}\left[\sigma^{-\sum_{i=1}^{n} U_{-i}}\right]
$$

In what follows we will focus on $\mathbb{E}[S]$, since analogous results for $\mathbb{E}[F]$ follow by replacing $\sigma$ with $\sigma^{-1}$ and $p$ with $1-p$. This follows from the stationarity of $\mathbf{U}$, which implies that for any $n$ the product $\sigma_{-1} \sigma_{-2} \cdots \sigma_{-n}$ has the same distribution as $\sigma_{1} \sigma_{2} \cdots \sigma_{n}$ (apply a shift over $n+1$ positions).

Consider now the RWRE swap model with a random environment generated by a Markov chain $\left\{Y_{i}, i \in \mathbb{Z}\right\}$, as specified in Section 2.2. Thus,

$$
\mathbb{E}[S]=\sum_{n=0}^{\infty} \mathbb{E}\left[\sigma^{\sum_{i=1}^{n} U_{i}}\right]=\sum_{n=0}^{\infty} \mathbb{E}\left[\sigma^{\sum_{i=1}^{n} g\left(Y_{i}\right)}\right] .
$$

For simplicity of notation, we assume that $M=\{1, \ldots, m\}$. Define

$$
G_{y}^{(n)}(\sigma)=\mathbb{E}\left[\sigma^{\sum_{i=1}^{n} g\left(Y_{i}\right)} \mid Y_{0}=y\right], \quad y=1, \ldots, m
$$

and let $P=\left(P_{y, y^{\prime}}\right)$ be the one-step transition matrix of $\left\{Y_{i}\right\}$. Then, by conditioning on $Y_{1}$,

$$
\begin{aligned}
G_{y}^{(n+1)}(\sigma) & =\mathbb{E}\left[\sigma^{\sum_{i=1}^{n+1} g\left(Y_{i}\right)} \mid Y_{0}=y\right]=\mathbb{E}\left[\sigma^{\sum_{i=2}^{n+1} g\left(Y_{i}\right)} \sigma^{g\left(Y_{1}\right)} \mid Y_{0}=y\right] \\
& =\sum_{y^{\prime}=1}^{m} P_{y, y^{\prime}} \sigma^{g\left(y^{\prime}\right)} G_{y^{\prime}}^{(n)}(\sigma) .
\end{aligned}
$$

In matrix notation, with $\mathbf{G}^{(n)}(\sigma)=\left(G_{1}^{(n)}(\sigma), \ldots, G_{m}^{(n)}(\sigma)\right)^{\top}$, we can write this as

$$
\mathbf{G}^{(n+1)}(\sigma)=P D \mathbf{G}^{(n)}(\sigma),
$$

where

$$
D=\operatorname{diag}\left(\sigma^{g(1)}, \ldots, \sigma^{g(m)}\right) .
$$


It follows, also using $G_{y}^{(0)}(\sigma)=1$, that

$$
\mathbf{G}^{(n)}(\sigma)=(P D)^{n} \mathbf{G}^{(0)}(\sigma)=(P D)^{n} \mathbf{1},
$$

where $\mathbf{1}=(1,1)^{\top}$, and hence

$$
\mathbb{E}[S]=\sum_{n=0}^{\infty} \pi \mathbf{G}^{(n)}(\sigma)=\pi \sum_{n=0}^{\infty}(P D)^{n} \mathbf{1}
$$

where $\boldsymbol{\pi}$ denotes the stationary distribution vector for $\left\{Y_{i}\right\}$. The matrix series $\sum_{n=0}^{\infty}(P D)^{n}$ converges if and only if $\operatorname{Sp}(P D)<1$, where $\operatorname{Sp}(\cdot)$ denotes the spectral radius, and in that case the limit is $(I-P D)^{-1}$, which leads to an explicit expression for $\mathbb{E}[S]$. We summarize these findings in the following theorem.

Theorem 3.1. For $\mathbb{E}\left[\ln \sigma_{0}\right]$ of Theorem 2.1 we have

$$
\mathbb{E}\left[\ln \sigma_{0}\right]=\ln \sigma \mathbb{E}\left[U_{0}\right]
$$

Thus, the a.s. limit of $X_{n}$ only depends on whether $p$ is less than or larger than 1/2, and on whether $\mathbb{E}\left[U_{0}\right]$ is positive or negative, regardless of the dependence structure between the $\left\{U_{i}\right\}$.

For $\mathbb{E}[S]$ of Theorem 2.2 we have, with matrices $P$ and $D$ as defined above

$$
\mathbb{E}[S]= \begin{cases}\boldsymbol{\pi}(I-P D)^{-1} \mathbf{1} & \text { if } S p(P D)<1 \\ \infty & \text { else. }\end{cases}
$$

Based on the above, the following subsections will give results on the transience/recurrence and on the drift for the random environments mentioned in Section 2.2. As will turn out, it is not trivial to determine when $\operatorname{Sp}(P D)<1$.

\subsection{Markov environment}

Recall that in this case $U_{i}=Y_{i}$, where $\left\{Y_{i}\right\}$ is a stationary discrete-time Markov chain on $\{-1,1\}$, with one-step transition matrix $P$ given by

$$
P=\left[\begin{array}{cc}
1-a & a \\
b & 1-b
\end{array}\right]
$$

for some $a, b \in(0,1)$. As a consequence, the quantity $\mathbb{E}\left[\ln \sigma_{0}\right]$ in Theorem 2.1, which determines whether $X_{n}$ will diverge to $+\infty$ or $-\infty$, or is recurrent, is given by

$$
\mathbb{E}\left[\ln \sigma_{0}\right]=\ln \sigma \mathbb{E}\left[U_{0}\right]=\ln \left(\frac{1-p}{p}\right) \frac{a-b}{a+b} .
$$

Hence, $X_{n} \rightarrow+\infty$ a.s. if and only if either $a>b$ and $p>1 / 2$, or $a<b$ and $p<1 / 2$; $X_{n} \rightarrow-\infty$ a.s. if and only if either $a>b$ and $p<1 / 2$, or $a<b$ and $p>1 / 2$; and $\left\{X_{n}\right\}$ is recurrent a.s. if and only if either $a=b$, or $p=1 / 2$, or both. 
Next, we study $\mathbb{E}[S]$ to find the drift. In the context of Section 3.1 the processes $\left\{U_{i}\right\}$ and $\left\{Y_{i}\right\}$ are identical and the function $g$ is the identity on the state space $\mathcal{U}=\{-1,1\}$. Thus, the matrix $D$ is given by $D=\operatorname{diag}\left(\sigma^{-1}, \sigma\right)$, and since $P$ is as (3.5), the matrix $P D$ is given by

$$
P D=\left[\begin{array}{cc}
(1-a) \sigma^{-1} & a \sigma \\
b \sigma^{-1} & (1-b) \sigma
\end{array}\right]
$$

for which we have the following.

Lemma 3.1. The matrix series $\sum_{n=0}^{\infty}(P D)^{n}$ converges to

$$
(I-P D)^{-1}=\frac{1}{\operatorname{det}(I-P D)}\left[\begin{array}{cc}
1-(1-b) \sigma & a \sigma \\
b \sigma^{-1} & 1-(1-a) \sigma^{-1}
\end{array}\right]
$$

with $\operatorname{det}(I-P D)=2-a-b-\left(\frac{1-a}{\sigma}+(1-b) \sigma\right)$, iff $1<\sigma<\frac{1-a}{1-b}$ (when $\left.a<b\right)$, or $\frac{1-a}{1-b}<\sigma<1$ (when $\left.a>b\right)$.

Proof. The series $\sum_{n=0}^{\infty}(P D)^{n}$ converges if and only if $\operatorname{Sp}(P D)<1$, where $\operatorname{Sp}(\cdot)$ denotes the spectral radius $\max _{i}\left|\lambda_{i}\right|$. The eigenvalues $\lambda_{1}, \lambda_{2}$ follow from

$$
|\lambda I-P D|=\lambda^{2}-A \lambda+(1-a-b)=0, \quad \text { where } \quad A=(1-a) \sigma^{-1}+(1-b) \sigma .
$$

The discriminant of this quadratic equation is

$$
A^{2}-4(1-a)(1-b)+4 a b=\left(\frac{1-a}{\sigma}-(1-b) \sigma\right)^{2}+4 a b>0,
$$

so the spectral radius is given by the largest eigenvalue,

$$
\operatorname{Sp}(P D)=\frac{A+\sqrt{A^{2}-4(1-a-b)}}{2} .
$$

Clearly $\operatorname{Sp}(P D)<1$ if and only if $\sqrt{A^{2}-4(1-a-b)}<2-A$, or equivalently $A<2-a-b$. Substituting the definition of $A$ and multiplying by $\sigma$ this leads to $(\sigma-1)((1-b) \sigma-(1-a))<0$. Since the coefficient of $\sigma^{2}$ in the above is $1-b>0$, the statement of the lemma now follows immediately.

This leads to the following proposition.

Proposition 3.1. We distinguish between transient cases with and without drift, and the recurrent case as follows.

1a. If either $a>b$ and $p \in\left(\frac{1}{2}, \frac{1-b}{(1-a)+(1-b)}\right)$ or $a<b$ and $p \in\left(\frac{1-b}{(1-a)+(1-b)}, \frac{1}{2}\right)$, then almost surely $\lim _{n \rightarrow \infty} X_{n}=\infty$ and

$$
V=(2 p-1) \frac{(1-b)(1-p)-(1-a) p}{\left(b+\frac{a-b}{a+b}\right)(1-p)+\left(a-\frac{a-b}{a+b}\right) p}>0 .
$$


1b. If either $a>b$ and $p \in\left(\frac{1-a}{(1-a)+(1-b)}, \frac{1}{2}\right)$ or $a<b$ and $p \in\left(\frac{1}{2}, \frac{1-a}{(1-a)+(1-b)}\right)$, then almost surely $\lim _{n \rightarrow \infty} X_{n}=-\infty$ and

$$
V=-(1-2 p) \frac{(1-b) p-(1-a)(1-p)}{\left(b+\frac{a-b}{a+b}\right) p+\left(a-\frac{a-b}{a+b}\right)(1-p)}<0 .
$$

2a. If either $a>b$ and $p \in\left[\frac{1-b}{(1-a)+(1-b)}, 1\right]$ or $a<b$ and $p \in\left[0, \frac{1-b}{(1-a)+(1-b)}\right]$, then almost surely $\lim _{n \rightarrow \infty} X_{n}=\infty$, but $V=0$.

2b. If either $a>b$ and $p \in\left[0, \frac{1-a}{(1-a)+(1-b)}\right]$ or $a<b$ and $p \in\left[\frac{1-a}{(1-a)+(1-b)}, 1\right]$, then almost surely $\lim _{n \rightarrow \infty} X_{n}=-\infty$, but $V=0$.

3. Otherwise (when $a=b$ or $p=1 / 2$ or both), $\left\{X_{n}\right\}$ is recurrent and $V=0$.

Proof. Substitution of (3.6) and $\boldsymbol{\pi}=\frac{1}{a+b}(b, a)$ into (3.4) leads to

$$
\begin{aligned}
V^{-1} & =2 \mathbb{E}[S]-1 \\
& =\frac{1+\sigma}{1-\sigma} \frac{\left(b+\frac{a-b}{a+b}\right) \sigma+\left(a-\frac{a-b}{a+b}\right)}{(1-b) \sigma-(1-a)} \\
& =\frac{1}{2 p-1} \frac{\left(b+\frac{a-b}{a+b}\right)(1-p)+\left(a-\frac{a-b}{a+b}\right) p}{(1-b)(1-p)-(1-a) p} .
\end{aligned}
$$

When $\sigma$ lies between 1 and $\frac{1-a}{1-b}$, i.e. when $p=(1+\sigma)^{-1}$ lies between $1 / 2$ and $(1-b) /((1-a)+(1-b))$, it follows by Lemma 3.1 that the process has positive drift, given by the reciprocal of the above. This proves (3.7). The proof of (3.8) follows from replacing $\sigma$ by $\sigma^{-1}$ and $p$ by $1-p$, and adding a minus sign. The other statements follow immediately.

Remark 3.1. When we take $a+b=1$ we obtain the case where the $\left\{U_{i}\right\}$ are iid with $\mathbb{P}\left(U_{i}=1\right)=\alpha=a /(a+b)$. In the following subsection we make a comparison between the Markov case and the iid case.

\subsubsection{Comparison with the iid environment}

To study the impact of the (Markovian) dependence, we reformulate the expression for the drift in Proposition 3.1 in terms of $\alpha=\mathbb{P}\left(U_{0}=1\right)=a /(a+b)$ and the correlation coefficient

$$
\varrho \equiv \varrho\left(U_{0}, U_{1}\right)=\frac{\operatorname{Cov}\left(U_{0}, U_{1}\right)}{\operatorname{Var}\left(U_{0}\right)}=\frac{\frac{a+b-4 a b}{a+b}-\left(\frac{a-b}{a+b}\right)^{2}}{1-\left(\frac{a-b}{a+b}\right)^{2}}=1-a-b .
$$

So $\varrho$ depends on $a$ and $b$ only through their sum $a+b$, with extreme values 1 (for $a=b=0$; i.e., $U_{i} \equiv U_{0}$ ) and -1 (for $a=b=1$; that is, $U_{2 i} \equiv U_{0}$ and 
$\left.U_{2 i+1} \equiv-U_{0}\right)$. The intermediate case $a+b=1$ leads to $\varrho=0$ and corresponds to the iid case. To express $V$ in terms of $\alpha$ and $\varrho$ we solve the system of equations $\frac{a}{a+b}=\alpha$ and $1-a-b=\varrho$, leading to the solution $a=(1-\varrho) \alpha$ and $b=(1-\varrho)(1-\alpha)$. Substitution into the expression for $V$ (here in the case of positive drift only, see (3.7)) and rewriting yields

$$
V=(2 p-1) \frac{\alpha-p+\varrho(1-\alpha-p)}{(\alpha(1-p)+(1-\alpha) p)(1+\varrho)-\varrho} .
$$

This enables us not only to immediately quickly obtain the drift for the iid case (take $\varrho=0$ ), but also to study the dependence of the drift $V$ on $\varrho$. Note that due to the restriction that $a$ and $b$ are probabilities, it must hold that $\varrho>\max \{1-$ $1 / \alpha, 1-1 /(1-\alpha)\}$.

Figures 2 illustrates various aspects of the difference between iid and Markov cases. Clearly, compared to the iid case (for the same value of $\alpha$ ), the Markov case with positive correlation coefficient has lower drift, but also a lower 'cutoff value' of $p$ at which the drift becomes zero. For negative correlation coefficients we see a higher cutoff value, but not all values of $\alpha$ are possible (since we should have $a<1$ ). Furthermore, for weak correlations the drift (if it exists) tends to be larger than for strong correlations (both positive and negative), depending on $p$ and $\alpha$.

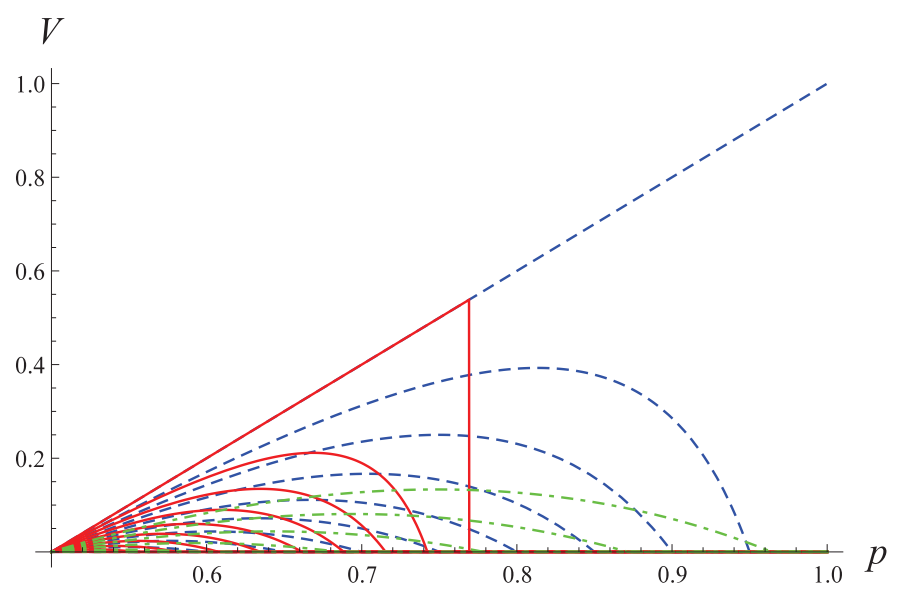

Figure 2: Drift for $\varrho=0$ (blue, dashed), $\varrho=0.3$ (red,solid), and $\varrho=-0.3$ (green, dotdashed) as a function of $p$. From highest to lowest curves for $\alpha=1,0.95, \ldots, 0.55$ (for $\varrho=0$ and $\varrho=0.3$ ), and for $\alpha=0.75,0.70, \ldots, 0.55$ (for $\varrho=-0.3$ ).

\section{$3.3 \quad 2$-dependent environment}

In this section we treat the $k$-dependent environment for $k=2$. For this case we have the transition probabilities

$$
P_{u_{i-2} u_{i-1}, u_{i}}=\mathbb{P}\left(U_{i}=u_{i} \mid U_{i-2}=u_{i-2}, U_{i-1}=u_{i-1}\right), \quad u_{j} \in\{-1,1\},
$$


so that the one-step transition matrix of the Markov chain $\left\{Y_{i}, i \in \mathbb{Z}\right\}$ with $Y_{i}=$ $\left(U_{i-1}, U_{i}\right)$ is given by

$P=\left[\begin{array}{cccc}P_{-1-1,-1} & P_{-1-1,+1} & 0 & 0 \\ 0 & 0 & P_{-1+1,-1} & P_{-1+1,+1} \\ P_{+1-1,-1} & P_{+1-1,+1} & 0 & 0 \\ 0 & 0 & P_{+1+1,-1} & P_{+1+1,+1}\end{array}\right]=\left[\begin{array}{cccc}1-a_{-} & a_{-} & 0 & 0 \\ 0 & 0 & b_{-} & 1-b_{-} \\ 1-a_{+} & a_{+} & 0 & 0 \\ 0 & 0 & b_{+} & 1-b_{+}\end{array}\right]$.

Thus, the model has five parameters, $a_{-}, a_{+}, b_{-}, b_{+}$, and $p$. Also note that the special case $a_{-}=a_{+}(=a)$ and $b_{-}=b_{+}(=b)$ corresponds to the (1-dependent) Markovian case in Section 3.2.

We first note that the stationary distribution (row) vector $\boldsymbol{\pi}$ is given by

$$
\boldsymbol{\pi}=\left(2+\frac{1-a_{+}}{a_{-}}+\frac{1-b_{-}}{b_{+}}\right)^{-1}\left(\frac{1-a_{+}}{a_{-}}, 1,1, \frac{1-b_{-}}{b_{+}}\right),
$$

so assuming stationarity we have $\mathbb{P}\left(U_{0}=1\right)=\pi_{-1,1}+\pi_{1,1}$ and $\mathbb{P}\left(U_{0}=-1\right)=$ $\pi_{-1,-1}+\pi_{1,-1}$. It follows that $\mathbb{P}\left(U_{0}=1\right)>\mathbb{P}\left(U_{0}=-1\right)$ if and only if $\frac{a_{-}}{1-a_{+}}>\frac{b_{+}}{1-b_{-}}$. This is important to determine the sign of $\mathbb{E}\left[\ln \sigma_{0}\right]$, which satisfies (with $\sigma=\frac{1-p}{p}$ as before),

$$
\mathbb{E}\left[\ln \sigma_{0}\right]=\left(2 \mathbb{P}\left(U_{0}=1\right)-1\right) \ln \sigma .
$$

Hence, $X_{n} \rightarrow+\infty$ a.s. if and only if either $\frac{a_{-}}{1-a_{+}}>\frac{b_{+}}{1-b_{-}}$and $p>1 / 2$, or $\frac{a_{-}}{1-a_{+}}<$ $\frac{b_{+}}{1-b_{-}}$and $p<1 / 2 ; X_{n} \rightarrow-\infty$ a.s. if and only if either $\frac{a_{-}}{1-a_{+}}>\frac{b_{+}}{1-b_{-}}$and $p<1 / 2$, or $\frac{a_{-}}{1-a_{+}}<\frac{b_{+}}{1-b_{-}}$and $p>1 / 2$; and $\left\{X_{n}\right\}$ is recurrent a.s. if and only if either $\frac{a_{-}}{1-a_{+}}=\frac{b_{+}}{1-b_{-}}$, or $p=1 / 2$, or both.

Next, we consider the drift. As before we have when $\mathbb{E}[S]<\infty$ that $V^{-1}=$ $2 \mathbb{E}[S]-1$. So in view of (3.4) we need to consider the matrix $P D$ where $D=$ $\operatorname{diag}\left(\sigma^{-1}, \sigma, \sigma^{-1}, \sigma\right)$, so

$$
P D=\left[\begin{array}{cccc}
\left(1-a_{-}\right) \sigma^{-1} & a_{-} \sigma & 0 & 0 \\
0 & 0 & b_{-} \sigma^{-1} & \left(1-b_{-}\right) \sigma \\
\left(1-a_{+}\right) \sigma^{-1} & a_{+} \sigma & 0 & 0 \\
0 & 0 & b_{+} \sigma^{-1} & \left(1-b_{+}\right) \sigma
\end{array}\right]
$$

and hence

$$
\begin{aligned}
V^{-1} & =2 \boldsymbol{\pi}\left(\sum_{n=0}^{\infty}(P D)^{n}\right) \mathbf{1}-1 \\
& =2 \boldsymbol{\pi}(I-P D)^{-1} \mathbf{1}-1
\end{aligned}
$$

if $\operatorname{Sp}(P D)<1$. Unfortunately, the eigenvalues of $P D$ are now the roots of a 4-degree polynomial, which are hard to find explicitly. However, using Perron-Frobenius theory and the implicit function theorem it is possible to prove the following lemma, which has the same structure as in the Markovian case. 
Lemma 3.2. The matrix series $\sum_{n=0}^{\infty}(P D)^{n}$ converges to $(I-P D)^{-1}$ iff $\sigma$ lies between 1 and $\frac{1-A}{1-B}$. Here, $A=a_{-}+a_{+} b_{-}-a_{-} b_{-}$and $B=b_{+}+a_{+} b_{-}-a_{+} b_{+}$.

Proof. To find out for which values of $\sigma$ we have $\operatorname{Sp}(P D)<1$, first we denote the (possibly complex) eigenvalues of $P D$ by $\lambda_{i}(\sigma), i=0,1,2,3$, as continuous functions of $\sigma$. Since $P D$ is a nonnegative irreducible matrix for any $\sigma>0$, we can apply Perron-Frobenius to claim that there is always a unique eigenvalue with largest absolute value (the other $\left|\lambda_{i}\right|$ being strictly smaller), and that this eigenvalue is real and positive (so in fact it always equals $\operatorname{Sp}(P D)$ ). When $\sigma=1$ the matrix is stochastic and we know this eigenvalue to be 1 , and denote it by $\lambda_{0}(1)$.

Now, moving $\sigma$ from 1 to any other positive value, $\lambda_{0}(\sigma)$ must continue to play the role of the Perron-Frobenius eigenvalue; i.e., none of the other $\lambda_{i}(\sigma)$ can at some point take over this role. If this were not true, then the continuity of the $\lambda_{i}(\sigma)$ would imply that one value $\widehat{\sigma}$ exists where (say) $\lambda_{1}$ 'overtakes' $\lambda_{0}$, meaning that $\left|\lambda_{1}(\widehat{\sigma})\right|=\left|\lambda_{0}(\widehat{\sigma})\right|$, which is in contradiction with the earlier Perron-Frobenius statement.

Thus, it remains to find out when $\lambda_{0}(\sigma)<1$, which can be established using the implicit function theorem, since $\lambda_{0}$ is implicitly defined as a function of $\sigma$ by $f\left(\sigma, \lambda_{0}\right)=0$, with $f(\sigma, \lambda)=\operatorname{det}(\lambda I-P D)$ together with $\lambda_{0}(1)=1$. Using $\operatorname{det}(D)=1$, we find that

$$
\begin{aligned}
f(\sigma, \lambda)= & \operatorname{det}\left(\left(\lambda D^{-1}-P\right) D\right)=\operatorname{det}\left(\lambda D^{-1}-P\right)= \\
= & \sigma\left[\lambda\left(a_{+} b_{-}-a_{+} b_{+}\right)+\lambda^{3}\left(b_{+}-1\right)\right] \\
& +\sigma^{-1}\left[\lambda\left(a_{+} b_{-}-a_{-} b_{-}\right)+\lambda^{3}\left(a_{-}-1\right)\right] \\
& +\lambda^{4}+\left(1-a_{-}-b_{+}+a_{-} b_{+}-a_{+} b_{-}\right) \lambda^{2}+a_{-} b_{-}-a_{-} b_{+}-a_{+} b_{-}+a_{+} b_{+} .
\end{aligned}
$$

Setting $\lambda=1$ in this expression gives $\operatorname{det}(I-P D)=-\sigma^{-1}(\sigma-1)((1-B) \sigma-(1-A))$, with two roots for $\sigma$. Thus, there is only an eigenvalue 1 when $\sigma=1$, which we already called $\lambda_{0}(1)$, or when $\sigma=\frac{1-A}{1-B}$. In the latter case this must be $\lambda_{0}\left(\frac{1-A}{1-B}\right)$, i.e., it cannot be $\lambda_{i}\left(\frac{1-A}{1-B}\right)$ for some $i \neq 0$, again due to continuity. As a result we have either $\lambda_{0}(\sigma)>1$ or $\lambda_{0}(\sigma)<1$ when $\sigma$ lies between 1 and $\frac{1-A}{1-B}$. Whether $\frac{1-A}{1-B}<1$ or $\frac{1-A}{1-B}>1$ depends on the parameters:

$$
\frac{1-A}{1-B}>1 \Leftrightarrow \frac{a_{-}}{1-a_{+}}<\frac{b_{+}}{1-b_{-}}
$$

where we used that $1-B=1-b_{+}-a_{+} b_{-}+a_{+} b_{+}>\left(1-b_{+}\right)\left(1-a_{+}\right)>0$. Now 
we apply the implicit function theorem:

$$
\begin{aligned}
\left.\frac{\mathrm{d} \lambda_{0}(\sigma)}{\mathrm{d} \sigma}\right|_{\sigma=1} & =-\left.\frac{\frac{\partial f\left(\sigma, \lambda_{0}\right)}{\partial \sigma}}{\frac{\partial f\left(\sigma, \lambda_{0}\right)}{\partial \lambda_{0}}}\right|_{\sigma=1, \lambda_{0}=1} \\
& =-\frac{b_{+}\left(1-a_{+}\right)-a_{-}\left(1-b_{-}\right)}{a_{-}\left(1-b_{-}+b_{+}\right)+b_{+}\left(1-a_{+}+a_{-}\right)} \\
& =\frac{\frac{a_{-}}{1-a_{+}}-\frac{b_{+}}{1-b_{-}}}{\frac{a_{-}}{1-a_{+}}\left(1+\frac{b_{+}}{1-b_{-}}\right)+\frac{b_{+}}{1-b_{-}}\left(1+\frac{a_{-}}{1-a_{+}}\right)},
\end{aligned}
$$

which due to (3.10) is $<0$ iff $\frac{1-A}{1-B}>1$ and is $>0$ iff $\frac{1-A}{1-B}<1$, so that indeed $\operatorname{Sp}(P D)=\lambda_{0}(\sigma)<1$ if and only if $\sigma$ lies between 1 and $\frac{1-A}{1-B}$.

Note that for the case $\frac{a_{-}}{1-a_{+}}=\frac{b_{+}}{1-b_{-}}$the series never converges, as there is no drift, $\mathbb{P}\left(U_{0}=1\right)=\mathbb{P}\left(U_{0}=-1\right)$. This corresponds to $a=b$ in the Markovian case and $\alpha=1 / 2$ in the iid case.

We conclude that if $\sigma$ lies between 1 and $\frac{1-A}{1-B}$, or equivalently, if $p$ lies between $1 / 2$ and $\frac{1-B}{1-A+1-B}$, the drift is given by $V=\left(2 \pi(I-P D)^{-1} \mathbf{1}-1\right)^{-1}$, where $\boldsymbol{\pi}$ is given in (3.9) and $(I-P D)^{-1}$ follows from Lemma 3.2. Using computer algebra, this can be shown to equal

$$
V=(2 p-1) \frac{d p(1-p)((1-B)(1-p)-(1-A) p)}{\sum_{i=0}^{3} c_{i} p^{i}}
$$

where

$$
\begin{aligned}
d & =a_{-}\left(b_{-}-b_{+}-1\right)+b_{+}\left(a_{+}-a_{-}-1\right) \\
c_{0} & =2 a_{-} b_{+}\left(b_{-}-b_{+}\right) \\
c_{1} & =-c_{0}\left(2+a_{+}+a_{-}\right)+(B-A)(1-B) \\
c_{2} & =-c_{0}-c_{1}-c_{3} \\
c_{3} & =(B-A)(2-A-B) .
\end{aligned}
$$

Including the transience/recurrence result from the first part of this section, and including the cases with negative drift, we obtain the following analogon to Proposition 3.1.

Proposition 3.2. We distinguish between transient cases with and without drift, and the recurrent case in the same way as for the Markov environment in Proposition 3.1. In particular, all statements (1a.), ..., (3) in Proposition 3.1 also hold for the 2-dependent environment if we replace $a$ and $b$ by $A$ and $B$ respectively, (3.7) by (3.14), and (3.8) by minus the same expression (3.14) but with $p$ replaced by $1-p$. 


\subsubsection{Comparison with the Markov environment}

To better observe the effect of the 2-dependence structure on the drift, it is convenient to reparameterize the model in terms of the parameters $\alpha=\mathbb{P}\left(U_{0}=1\right), \varrho_{01}=$ $\varrho\left(U_{0}, U_{1}\right), \varrho_{02}=\varrho\left(U_{0}, U_{2}\right)$ (correlation coefficients), and $e_{012}=\mathbb{E}\left[U_{0} U_{1} U_{2}\right]$; for more details see [12]. Note that due to the restriction that $a_{-}, a_{+}, b_{-}$, and $b_{+}$are probabilities, $\left(\alpha, \varrho_{01}, \varrho_{02}, e_{012}\right)$ can only take values in a strict subset of $[0,1] \times[-1,1]^{3}$.

Figure 3 illustrates the significant differences in drift behaviors for the Markovian, independent and various 2 -dependent cases, all with the same $\alpha=0.95$ and $\varrho_{1}=0.3$. Note that the the cutoff value for the Markovian case is here approximately 0.75 . By varying $\varrho_{2}$ and $e_{012}$ one can achieve a considerable increase in the drift. It is not too difficult to verify that the smallest possible value for $\varrho_{2}$ is here $(\alpha-1) / \alpha=-1 / 19$, in which case $e_{012}$ can only take the value $3+2 \alpha\left(-5-4 \alpha\left(-1+\varrho_{1}\right)+4 \varrho_{1}\right)=417 / 500$. This gives a maximal cutoff value of 1 . The corresponding drift curve is indicated by the "maximal" label in Figure 3. For $\varrho_{2}=0$, the parameter $e_{012}$ can at most vary from $-1+2 \alpha\left(-1+\alpha\left(2-4 \varrho_{1}\right)+4 \varrho_{1}\right)=$ $103 / 123=0.824$ to $7+2 \alpha\left(-9+\alpha\left(6-4 \varrho_{1}\right)+4 \varrho_{1}\right)=211 / 250=0.844$. The solid red curves show the evolution of the drift between these extremes. The dashed blue curve corresponds to the drift for the independent case with $\alpha=0.95$.

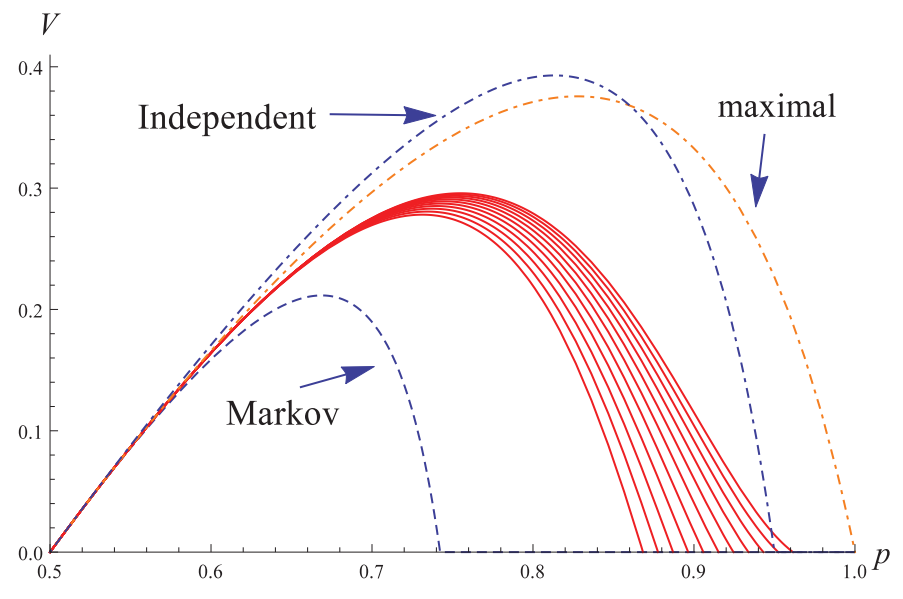

Figure 3: Drift for $\alpha=0.95$ and $\varrho_{1}=0.3$ for various $\varrho_{2}$ and $e_{012}$. The solid red curves show the drift for $\varrho_{2}=0$ and $e_{012}$ varying from 0.824 to 0.844 . The smallest dashed blue curve corresponds to the Markov case. The "maximal" dotdashed orange curve corresponds to the case $\varrho_{2}=-1 / 19$ and $e_{012}=417 / 500$. The middle dashed blue line gives the independent case.

\subsection{Moving average environment}

Recall that the environment is given by $U_{i}=g\left(Y_{i}\right)$ where the Markov process $\left\{Y_{i}\right\}$ is given by $Y_{i}=\left(\widehat{U}_{i}, \widehat{U}_{i+1}, \widehat{U}_{i+2}\right)$. The sequence $\left\{\widehat{U}_{i}\right\}$ is iid with $\mathbb{P}\left(\widehat{U}_{i}=1\right)=\alpha=$ 
$1-\mathbb{P}\left(\widehat{U}_{i}=-1\right)$. Thus, $\left\{Y_{i}\right\}$ has states $1=(-1,-1,-1), 2=(-1,-1,1), \ldots, 8=$ $(1,1,1)$ (in lexicographical order) and transition matrix $P$ given by $(2.7)$. The deterministic function $g$ is given by (2.8).

The almost sure behavior of $\left\{X_{n}\right\}$ again depends only on $\mathbb{E}\left[U_{0}\right]$ which equals $-4 \alpha^{3}+6 \alpha^{2}-1=(2 \alpha-1)\left(-2 \alpha^{2}+2 \alpha+1\right)$. Since $-2 \alpha^{2}+2 \alpha+1>0$ for $0 \leqslant \alpha \leqslant 1$, the sign of $\mathbb{E}\left[U_{0}\right]$ is the same as the sign of $\mathbb{E}\left[\widehat{U}_{0}\right]=2 \alpha-1$, so the almost sure behavior is precisely the same as in the iid case; we will not repeat it here (but see Proposition 3.3).

To study the drift, we need the stationary vector of $\left\{Y_{i}\right\}$, which is given by

$$
\begin{array}{r}
\boldsymbol{\pi}=\left\{(1-\alpha)^{3},(1-\alpha)^{2} \alpha,(1-\alpha)^{2} \alpha,(1-\alpha) \alpha^{2},\right. \\
\left.(1-\alpha)^{2} \alpha,(1-\alpha) \alpha^{2},(1-\alpha) \alpha^{2}, \alpha^{3}\right\},
\end{array}
$$

and the convergence behavior of $\sum(P D)^{n}$, with $D=\operatorname{diag}\left(\sigma^{-1}, \sigma^{-1}, \sigma^{-1}, \sigma, \sigma^{-1}\right.$, $\sigma, \sigma, \sigma)$. This is given in the following lemma.

Lemma 3.3. The matrix series $\sum_{n=0}^{\infty}(P D)^{n}$ converges to $(I-P D)^{-1}$ iff $\sigma$ lies between 1 and $\sigma_{\text {cutoff }}$, which is the unique root $\neq 1$ of

$$
\begin{aligned}
\operatorname{det}(I-P D)= & -\frac{\alpha(1-\alpha)^{2}}{\sigma^{3}}+\frac{\alpha^{2}(1-\alpha)^{2}}{\sigma^{2}}-\frac{(1-\alpha)\left(1-\alpha+\alpha^{2}\right)}{\sigma}+1 \\
& -2 \alpha^{2}(1-\alpha)^{2}-\alpha^{2}(1-\alpha) \sigma^{3}+\alpha^{2}(1-\alpha)^{2} \sigma^{2}-\alpha\left(1-\alpha+\alpha^{2}\right) \sigma .
\end{aligned}
$$

Proof. The proof is similar to that of Lemma 3.2; we only give an outline, leaving details for the reader to verify. Again, denote the possibly complex eigenvalues of $P D$ by $\lambda_{i}(\sigma), i=0, \ldots, 7$ and use Perron-Frobenius theory to conclude that for any $\sigma>0$ we have $\operatorname{Sp}(P D)=\lambda_{0}(\sigma)$, say, with $\lambda_{0}(1)=1$.

To find out when $\lambda_{0}(\sigma)<1$ we again use the implicit function theorem on $f\left(\sigma, \lambda_{0}\right)=0$, with $f(\sigma, \lambda)=\operatorname{det}(\lambda I-P D)$. Setting $\lambda=1$ gives (3.16). It can be shown that $f(\sigma, 1)$ is zero at $\sigma=1$, that $f(\sigma, 1) \rightarrow \infty$ for $\sigma \downarrow 0$, and that $\left(\partial^{2} / \partial \sigma^{2}\right) f(\sigma, 1)<0$ for all $\sigma>0$ (for the latter, consider $0<\sigma<1$ and $\sigma \geqslant 1$ separately). Thus we can conclude that $f(\sigma, 1)$ has precisely two roots for $\sigma>0$, at $\sigma=1$ and at $\sigma=\sigma_{\text {cutoff }}$.

As a result we have either $\lambda_{0}(\sigma)>1$ or $\lambda_{0}(\sigma)<1$ when $\sigma$ lies between 1 and $\sigma_{\text {cutoff }}$. For the location of $\sigma_{\text {cutoff }}$ it is helpful to know that $\left.(\partial / \partial \sigma) f(\sigma, 1)\right|_{\sigma=1}=$ $(2 \alpha-1)\left(2 \alpha^{2}-2 \alpha-1\right)$, which is positive for $0<\alpha<1 / 2$ and negative for $1 / 2<$ $\alpha<1$. Thus we have $\sigma_{\text {cutoff }}>1$ iff $\alpha<1 / 2$. Also $(\partial / \partial \lambda) f(1,1)=1$ so that the implicit function theorem gives $\left.(d / d \sigma) \lambda_{0}(\sigma)\right|_{\sigma=1}=-(2 \alpha-1)\left(2 \alpha^{2}-2 \alpha-1\right)$, so that indeed $\lambda_{0}(\sigma)<1$ iff $\sigma$ lies between 1 and $\sigma_{\text {cutoff }}$. 
The cutoff value for $p$ is now easily found as $\left(1+\sigma_{\text {cutoff }}\right)^{-1}$, which can be numerically evaluated. The values are plotted in Figure 4.

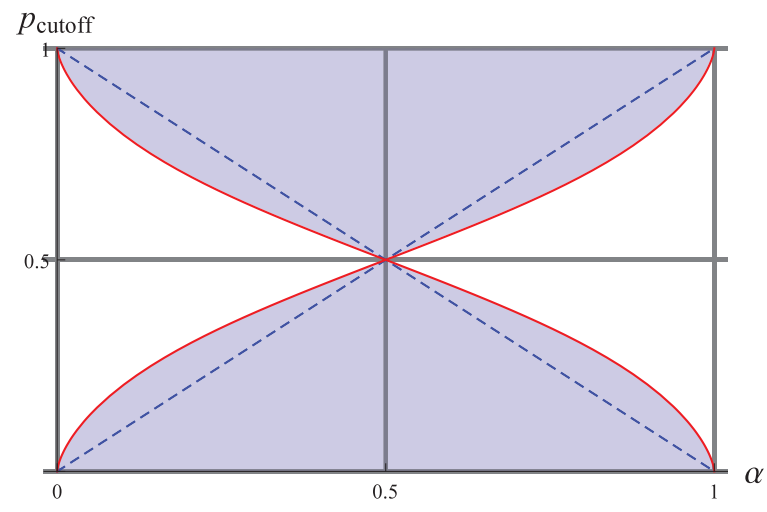

Figure 4: Relation between cutoff value for $p$, and $\alpha$. In the left (right) white region the drift is stricty positive (negative). In the shaded region the drift is 0 . The solid red curve is for the moving average process. For comparison, the dashed blue line is the iid case.

When $p$ lies between $1 / 2$ and $p_{\text {cutoff }}$, the drift is given by $V=\left(2 \pi(I-P D)^{-1} \mathbf{1}-\right.$ $1)^{-1}$, where $\boldsymbol{\pi}$ is given in (3.15) and $(I-P D)^{-1}$ follows from Lemma 3.3. Using computer algebra we can find a rather unattractive, but explicit expression for the value of the drift; it is given by the quotient of

$$
\begin{aligned}
& \alpha^{4}\left(-(1-2 p)^{2}\right)(p-1) p+\alpha^{3}(1-2 p((p-2) p(p(2 p-5)+6)+4)) \\
& +\alpha^{2}(2 p-1)(p(3 p((p-2) p+3)-5)+1)-\alpha(1-2 p)^{2} p^{2}-(p-1)^{2} p^{3}(2 p-1)
\end{aligned}
$$

and

$$
\begin{aligned}
& -2 \alpha^{5}(2 p-1)^{3}-\alpha^{4}(1-2 p)^{2}((p-11) p+6)+\alpha^{3}(2 p-1)\left(2 p\left(p^{3}-9 p+10\right)\right. \\
& -5)-\alpha^{2}(p+1)(2 p-1)(p(p(3 p-7)+6)-1)+\alpha p^{2}(2 p-1)+(p-1)^{2} p^{3} .
\end{aligned}
$$

Proposition 3.3. Let $p_{\text {cutoff }}=\left(1+\sigma_{\text {cutoff }}\right)^{-1}$, where $\sigma_{\text {cutoff }}$ follows from Lemma 3.3. Then $p_{\text {cutoff }}>1 / 2$ iff $\alpha>1 / 2$. We distinguish between transient cases with and without drift, and the recurrent case as follows.

1a. If either $\alpha>1 / 2$ and $p \in\left(1 / 2, p_{\text {cutoff }}\right)$ or $\alpha<1 / 2$ and $p \in\left(p_{\text {cutoff }}, 1 / 2\right)$, then almost surely $\lim _{n \rightarrow \infty} X_{n}=\infty$ and the drift $V>0$ is given as above.

1b. If either $\alpha>1 / 2$ and $p \in\left(1-p_{\text {cutoff }}, 1 / 2\right)$ or $\alpha<1 / 2$ and $p \in\left(1 / 2,1-p_{\text {cutoff }}\right)$, then almost surely $\lim _{n \rightarrow \infty} X_{n}=-\infty$ and the drift $V<0$ is given as minus the same expression as above but with $p$ replaced by $1-p$.

2a. If either $\alpha>1 / 2$ and $p \in\left[p_{\text {cutoff }}, 1\right]$ or $\alpha<1 / 2$ and $p \in\left[0, p_{\text {cutoff }}\right]$, then almost surely $\lim _{n \rightarrow \infty} X_{n}=\infty$, but $V=0$. 
2b. If either $\alpha>1 / 2$ and $p \in\left[0,1-p_{\text {cutoff }}\right]$ or $\alpha<1 / 2$ and $p \in\left[1-p_{\text {cutoff }}, 1\right]$, then almost surely $\lim _{n \rightarrow \infty} X_{n}=-\infty$, but $V=0$.

3. Otherwise (when $\alpha=1 / 2$ or $p=1 / 2$ or both), $\left\{X_{n}\right\}$ is recurrent and $V=0$.

Figure 5 compares the drifts for the moving average and independent environments.

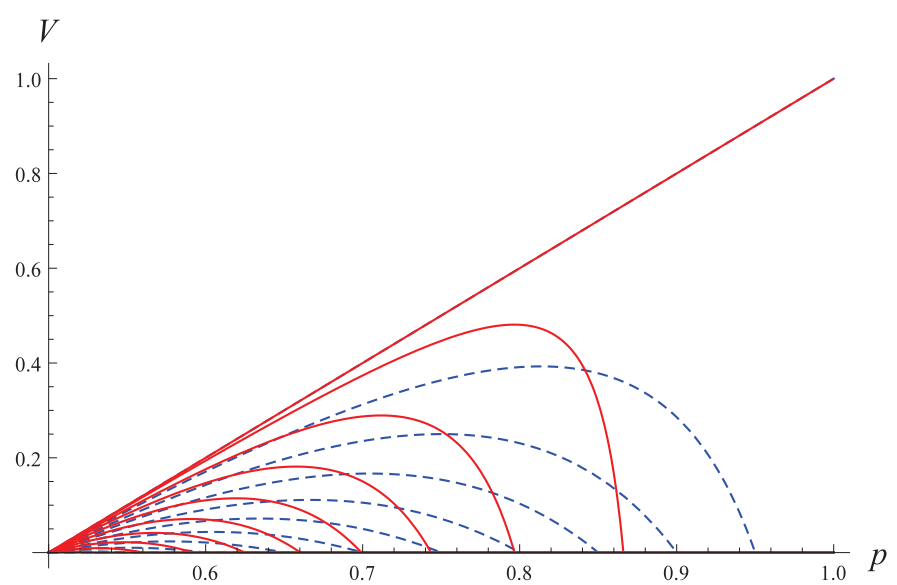

Figure 5: Red: Drift for the moving average environment as a function of $p$ for $\alpha=1,0.95, \ldots, 0.55$ (from highest to lowest curves). Blue: comparison with the independent case.

It is interesting to note that the cutoff points (where $V$ becomes 0 ) are significantly lower in the moving average case than the iid case, using the same $\alpha$, while at the same time the maximal drift that can be achieved is higher for the moving average case than for the iid case. This is different behavior from the Markovian case; see also Figure 2.

\section{Conclusions}

Random walks in random environments can exhibit interesting and unusual behavior due to the trapping phenomenon. The dependency structure of the random environment can significantly affect the drift of the process. We showed how to conveniently construct dependent environment processes, including $k$-dependent and moving average environments, by using an auxiliary Markov chain. For the wellknown swap RWRE model, this approach allows for easy computation of drift, as well as explicit conditions under which the drift is positive, negative, or zero. The cutoff values where the drift becomes zero, are determined via Perron-Frobenius theory. Various generalizations of the above environments can be considered in the same (swap model) framework, and can be analyzed along the same lines, e.g., 
replacing iid by Markovian $\left\{\widehat{U}_{i}\right\}$ in the moving average model, or taking moving averages of more than 3 neighboring states.

Other possible directions for future research are (a) extending the two-state dependent random environment to a $k$-state dependent random environment; (b) replacing the transition probabilities for swap model with the more general rules in Eq.(2.1); and (c) generalizing the single-state random walk process to a multistate discrete-time quasi birth and death process (see, e.g., [2]). By using an infinite "phase space" for such processes, it might be possible to bridge the gap between the theory for one- and multi-dimensional RWREs.

\section{Acknowledgements}

This work was supported by the Australian Research Council Centre of Excellence for Mathematical and Statistical Frontiers (ACEMS) under grant number CE140100049. Part of this work was done while the first author was an Ethel Raybould Visiting Fellow at The University of Queensland.

\section{References}

[1] S. Alili. Asymptotic behaviour for random walks in random environments. $J$. Appl. Prob., 36:334-349, 1999.

[2] N. G. Bean, L. Bright, G. Latouche, C. E. M. Pearce, P. K. Pollett, and P. G. Taylor. The quasi-stationary behavior of quasi-birth-and-death processes. The Annals of Applied Probability, 7(1):134-155, 021997.

[3] T. Brereton, D.P. Kroese, O. Stenzel, V. Schmidt, and B. Baumeier. Efficient simulation of charge transport in deep-trap media. In C. Laroque, J. Himmelspach, R. Pasupathy, O. Rose, and A. M. Uhrmacher, editors, Proceedings of the 2012 Winter Simulation Conference, Berlin, 2012.

[4] A. A. Chernov. Replication of multicomponent chain by the lighting mechanism. Biophysics, 12:336-341, 1967.

[5] D. Dolgopyat, G. Keller, and C. Liverani. Random walk in Markovian environment. The Annals of Probability, 36(5):1676-1710, 092008.

[6] A. Greven and F. den Hollander. Large deviations for a random walk in random environment. Ann. Probab., 22:1381-1428, 1994.

[7] B. D. Hughes. Random Walks and Random Environments. Oxford University Press, 1996. 
[8] H. Kesten, M. W. Koslow, and F. Spitzer. A limit law for random walk in a random environment. Compositio Math., pages 145-168, 1975.

[9] S. M. Kozlov. The method of averaging and walks in inhomogeneous enviroments. Russian Math. Surveys, 40:73-145, 1985.

[10] E. Mayer-Wolf, A. Roitershtein, and O. Zeitouni. Limit theorems for onedimensional transient random walks in Markov environments. Ann. Inst. H. Poincaré Probab. Statist., 40(5):635-659, 2004.

[11] P. Révész. Random Walk in Random and Non-Random Environments. World Scientific, third edition, 2013.

[12] R. W. R. Scheinhardt and D. P. Kroese. Computing the drift of random walks in dependent random environments. arXiv:1406.3390v1, 2014.

[13] Y. G. Sinai. The limiting behavior of a one-dimensional random walk in a random medium. Theory Prob. Appl., 27(2):256-268, 1982.

[14] F. Solomon. Random walks in a random environment. Ann. Prob., 3:1-31, 1975.

[15] O. Stenzel, C. Hirsch, V. Schmidt, T. Brereton T., D.P. Kroese, B. Baumeier, and D. Andrienko. A general framework for consistent estimation of charge transport properties via random walks in random environments. Multiscale Modeling and Simulation, 2014. Accepted for publication, pending minor revision.

[16] A.-S. Sznitman. Topics in random walks in random environment, pages 203266. ICTP Lecture Notes Series, Trieste, 2004.

[17] D. E. Temkin. A theory of diffusionless crystal growth. Kristallografiya, 14:423$430,1969$.

[18] O. Zeitouni. Lecture notes on random walks in random environment, volume 1837 of Lecture Notes in Mathematics. Springer, 2004.

[19] O. Zeitouni. Random walks in random environment. In Robert A. Meyers, editor, Computational Complexity, pages 2564-2577. Springer, New York, 2012. 